\title{
Lie Group Analysis of Heat and Mass Transfer Effects on Steady MHD Free Convection Flow past an Inclined Surface with Viscous Dissipation
}

\author{
M. G. Reddy \\ Department of Mathematics, Acharya Nagarjuna University Ongole Campus, Ongole,A.P. (India) - 523001 \\ mgrmaths@gmail.com
}

(Received December 11, 2011; accepted June 25, 2012)

\begin{abstract}
This article concerns with a steady two-dimensional flow of an electrically conducting incompressible dissipating fluid over an inclined semi-infinite surface with heat and mass transfer. The flow is permeated by a uniform transverse magnetic field. A scaling group of transformations is applied to the governing equations. The system remains invariant due to some relations among the parameters of the transformations. After finding three absolute invariants, a third-order ordinary differential equation corresponding to the momentum equation, and two secondorder ordinary differential equations corresponding to energy and diffusion equations are derived. The coupled ordinary differential equations along with the boundary conditions are solved numerically. Comparisons with previously published work are performed and the results are found to be in very good agreement. Many results are obtained and a representative set is displayed graphically to illustrate the influence of the various parameters on the dimensionless velocity, temperature and concentration profiles. It is found that the velocity increases with an increase in the thermal and solutal Grashof numbers. The velocity and concentration of the fluid decreases with an increase in the Schmidt number. The results, thus, obtained are presented graphically and discussed.
\end{abstract}

Keywords: Lie group analysis, Natural convection, MHD, Viscous dissipation, Heat and mass transfer, Inclined surface

\section{NOMENCLATURE}

applied magnetic field

species concentration in the boundary layer

the species concentration in the fluid far away from the plate

specific heat at constant pressure

mass diffusivity

Eckert number

dimensionless stream function

acceleration due to gravity

local temperature Grashof number

local mass Grashof number

thermal conductivity of the fluid

magnetic field parameter

Prandtl number

Schmidt number

temperature of the fluid in the boundary layer
$T_{\infty} \quad$ the temperature of the fluid in the boundary layer

$u, v \quad$ velocity components in $x, y$ directions

$\eta \quad$ similarity variable

$\alpha \quad$ angle of inclination

$\beta \quad$ coefficient of thermal expansion

$\beta^{*} \quad$ coefficient of concentration expansion

$\sigma \quad$ electrical conductivity

$\rho$ density of the fluid

$v \quad$ kinematic viscosity

$\theta$ dimensionless temperature

$\phi$ dimensionless concentration

$w \quad$ condition at wall

$\infty \quad$ condition at infinity

()$^{\prime} \quad$ differentiation with respect to $\eta$ 


\section{INTRODUCTION}

The study of natural convection flow for an incompressible viscous fluid past a heated surface has attracted the interest of many researchers in view of its important applications to many engineering problems such as cooling of nuclear reactors, the boundary layer control in aerodynamics, crystal growth, food processing and cooling towers. In this paper, symmetry methods are applied to a natural convection boundary layer problem. The main advantage of such methods is that they can successfully be applied to non-linear differential equations. The symmetries of differential equations are those continuous groups of transformations under which the differential equations remain invariant, that is, a symmetry group maps any solution to another solution. The symmetry solutions are quite popular because they result in the reduction of the number of independent variables of the problem. Chen (2004) performed an analysis to study the natural convection flow over a permeable inclined surface with variable wall temperature and concentration. The results show that the velocity is decreased in the presence of a magnetic field. Increasing the angle of inclination decreases the effect of buoyancy force. Heat transfer rate is increased when the Prandtl number is increased. Ibrahim et al. (2005) investigated the similarity reductions for problems of radiative and magnetic field effects on free convection and mass-transfer flow past a semi-infinite flat plate. They obtained new similarity reductions and found an analytical solution for the uniform magnetic field by using Lie group method. They also presented the numerical results for the nonuniform magnetic field.

Hydro magnetic incompressible viscous flow has many important engineering applications such as magneto hydrodynamic power generators and the cooling of reactors also its applications to problems in geophysics, astrophysics etc. The study of magnetohydrodynamics (MHD) plays an important role in agriculture, engineering and petroleum industries. The problem of free convection under the influence of a magnetic field has attracted the interest of many researchers in view of its applications in geophysics and astrophysics. Soundalgekar et al. (1979) analyzed the problem of free convection effects on Stokes problem for a vertical plate under the action of transversely applied magnetic field. Elbashbeshy (1997) studied the heat and mass transfer along a vertical plate under the combined buoyancy effects of thermal and species diffusion, in the presence of magnetic field. Helmy (1998) presented an unsteady two-dimensional laminar free convection flow of an incompressible, electrically conducting (Newtonian or polar) fluid through a porous medium bounded by an infinite vertical plane surface of constant temperature.

Kalpadides and Balassas (2004) studied the free convective boundary layer problem of an electrically conducting fluid over an elastic surface by group theoretic method. Their results agreed with the existing result for the group of scaling symmetry. They found that the numerical solution also does so. The NavierStokes and boundary layer equations for incompressible flows were derived using a convenient coordinate system by Pakdemirli (1992). The results showed that the boundary layer equations accept similarity solutions for the constant pressure gradient case. The importance of similarity transformations and their applications to partial differential equations was studied by Pakdemirli and Yurusoy (1998). They investigated the special group transformations for producing similarity solutions. They also discussed spiral group of transformations. Using Lie group analysis, three dimensional, unsteady, laminar boundary layer equations of non-Newtonian fluids are studied by Yurusoy and Pakdemirli (1997, 1999). They assumed that the shear stresses are arbitrary functions of the velocity gradients. Using Lie group analysis, they obtained two different reductions to ordinary differential equations. They also studied the effects of a moving surface with vertical suction or injection through the porous surface. They further studied exact solution of boundary layer equations of a special nonNewtonian fluid overa stretching sheet by the method of Lie group analysis. They found that the boundary layer thickness increases when the non-Newtonian behaviour increases. They also compared the results with that for a Newtonian fluid. Yurusoy and Pakdemirli (2001) investigated the Lie group analysis of creeping flow of a second grade fluid. They constructed an exponential type of exact solution using the translation symmetry and a series type of approximate solution using the scaling symmetry.

Viscous mechanical dissipation effects are important in geophysical flows and also in certain industrial operations and are usually characterized by the Eckert number. In most of the studies mentioned above, viscous dissipation is neglected. Gebhart (1962) reported the influence of viscous heating dissipation effects in natural convective flows, showing that the heat transfer rates are reduced by an increase in the dissipation parameter. Gebhart and Mollendorf (1969) considered the effects of viscous dissipation for the external natural convection flow over a surface.

Combined heat and mass transfer problems of importance in many processes and have, therefore, received a considerable amount of attention in recent years. In processes such as drying, evaporation at the surface of a water body, energy transfer in a wet cooling tower and the flow in a desert cooler, heat and mass transfer occur simultaneously. Possible applications of this type of flow can be found in many industries. For example, in the power industry, among the methods of generating electric power is one in which electrical energy is extracted directly from a moving conducting fluid. Gnaneswara Reddy and Bhaskar Reddy (2010) studied the radiation and mass transfer effects on unsteady MHD free convection flow past a vertical porous plate with viscous dissipation by using finite element method. Recently, Gnaneswara Reddy and Bhaskar Reddy(2011) investigated mass transfer and heat generation effects on MHD free convection flow past an inclined vertical surface in a porous medium. Sivasankaran et al. (2006) analyzed lie group analysis of natural convection heat and mass transfer in an inclined surface. Gnaneswara Reddy and Bhaskar Reddy(2010) have presented soret and dufour effects on steady MHD free convection flow past a semi-infinite moving vertical plate in a porous medium with viscous dissipation. 
In this article, application of scaling group of transformation for Heat and mass transfer effects on steady free convection flowin an inclined plate in the presence of MHD and viscous dissipation has been employed. This reduces the system of nonlinear coupled partial differential equations governing the motion of fluid into a system of coupled ordinary differential equations by reducing the number of independent variables. The system remains invariant due to some relations among the parameters of the transformations. Three absolute invariants are obtained and used to derive a third-order ordinary differential equation corresponding to momentum equation and two second-order ordinary differential equations corresponding to energy and diffusion equations. With the use of Runge-Kutta fourth order along shooting method, the equations are solved. Finally, analysis has been made to investigate the effects of thermal and solutalGrashof numbers, magnetic field parameter, Prandtl number, Viscous dissipation parameter, and Schmidt number on the motion of fluid using scaling group of transformations, viz., Lie group transformations.

\section{MATHEMATICAL ANALYSIS}

Consider the heat and mass transfer of a steady twodimensional hydromagnetic flow of a viscous, incompressible, electrically conducting and dissipating fluid past a semi-infinite inclined plate with an acute angle ${ }^{\alpha}$ to the vertical. The flow is assumed to be in the ${ }^{x}$ - direction, which is taken along the semi-infinite inclined plate and $y_{-}$axis normal to it. The flow configuration and coordinate system are shown in Fig.1.

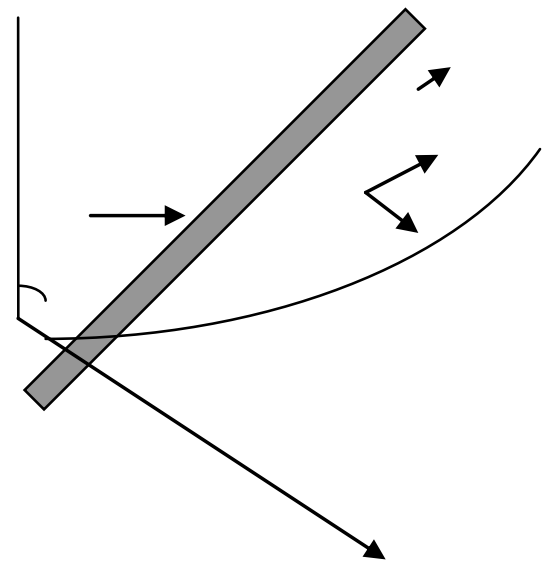

Fig. 1. Flow configuration and coordinate system.

A magnetic field of uniform strength $B_{0}$ is introduced normal to the direction of the flow. In the analysis, we assume that the magnetic Reynolds number is much less than unity so that the induced magnetic field is neglected in comparison to the applied magnetic field. It is also assumed that all fluid properties are constant except that of the influence of the density variation with temperature and concentration in the body force term. The surface is maintained at a constant temperature $T_{w}$, which is higher than the constant temperature $T_{\infty}$ of the surrounding fluid and the concentration $C_{w}$ is greater than the constant concentration $C_{\infty}$. The level of concentration of foreign mass is assumed to be low, so that the Soret and Dufour effects are negligible. Then, under the usual Boussinesq's and boundary layer approximations, the governing equations are

Continuity equation

$\frac{\partial u}{\partial x}+\frac{\partial u}{\partial y}=\mathbf{0}$

Momentum equation

$$
\begin{array}{r}
u \frac{\partial u}{\partial x}+v \frac{\partial u}{\partial y}=v \frac{\partial^{2} u}{\partial y^{2}}+g \beta\left(T-T_{\infty}\right) \cos \alpha \\
+g \beta^{*}\left(C-C_{\infty}\right) \cos \alpha-\frac{\sigma B_{0}^{2}}{\rho} u
\end{array}
$$

Energy equation

$u \frac{\partial T}{\partial x}+v \frac{\partial T}{\partial y}=\frac{k}{\rho c_{p}} \frac{\partial^{2} T}{\partial y^{2}}+\frac{\mu}{\rho c_{p}}\left(\frac{\partial u}{\partial y}\right)^{2}$

Species equation

$u \frac{\partial C}{\partial x}+\mathrm{v} \frac{\partial C}{\partial y}=\mathrm{D} \frac{\partial^{2} C}{\partial y^{2}}$

The boundary conditions for the velocity, temperature and concentration fields are

$u=v=0, T=T_{w}, C=C_{w}$ at $y=0$
$u \rightarrow 0, T \rightarrow T_{\infty}, C \rightarrow C_{\infty}$ as $y \rightarrow \infty$

On introducing the following non-dimensional quantities

$$
\begin{aligned}
& \bar{x}=\frac{x U_{\infty}}{v}, \bar{y}=\frac{y U_{\infty}}{v}, \bar{u}=\frac{u}{U_{\infty}}, \quad \bar{v}=\frac{v}{U_{\infty}}, \\
& M=\frac{\sigma B_{0}^{2} v}{U_{\infty}^{3}}, G r=\frac{v g \beta\left(T_{w}-T_{\infty}\right)}{U_{\infty}^{3}}, \theta=\frac{T-T_{\infty}}{T_{w}-T_{\infty}}, \\
& G m=\frac{v g \beta^{*}\left(C_{w}-C_{\infty}\right)}{U_{\infty}^{3}}, \phi=\frac{C-C_{\infty}}{C_{w}-C_{\infty}}, \\
& \operatorname{Pr}=\frac{v}{\alpha}, \quad E c=\frac{U_{\infty}^{2}}{c_{p}\left(T_{w}-T_{\infty}\right)}, S c=\frac{v}{D}
\end{aligned}
$$

Substituting Eq. (6) into Eqs. (1) - (4) and dropping the bars, we obtain,

$$
\frac{\partial u}{\partial x}+\frac{\partial v}{\partial y}=0
$$

$$
\begin{aligned}
u \frac{\partial u}{\partial x}+v \frac{\partial u}{\partial y} & =\frac{\partial^{2} u}{\partial y^{2}}+\operatorname{Gr} \theta \operatorname{Cos} \alpha \\
& +G m \phi \operatorname{Cos} \alpha-M u
\end{aligned}
$$


M. G. Reddy / JAFM, Vol. 6, No. 3, pp. 397-404, 2013.

$$
\begin{aligned}
& u \frac{\partial \theta}{\partial x}+v \frac{\partial \theta}{\partial y}=\frac{1}{\operatorname{Pr}} \frac{\partial^{2} \theta}{\partial y^{2}}+E c\left(\frac{\partial u}{\partial y}\right)^{2} \\
& u \frac{\partial \phi}{\partial x}+v \frac{\partial \phi}{\partial y}=\frac{1}{S c} \frac{\partial^{2} \phi}{\partial y^{2}}
\end{aligned}
$$

The corresponding boundary conditions take the form

$$
\begin{aligned}
& u=v=0, \quad \theta=1, \phi=1 \text { at } y=0 \\
& u \rightarrow 0, \theta \rightarrow 0, \phi \rightarrow 0 \text { as } y \rightarrow \infty
\end{aligned}
$$

By using the stream function $u=\frac{\partial \psi}{\partial y}, v=-\frac{\partial \psi}{\partial x}$ we have

$$
\begin{gathered}
\left(\frac{\partial \psi}{\partial y} \frac{\partial^{2} \psi}{\partial x \partial y}-\frac{\partial \psi}{\partial x} \frac{\partial^{2} \psi}{\partial y^{2}}\right)=\frac{\partial^{3} \psi}{\partial y^{3}}+G r \theta \operatorname{Cos} \alpha \\
+G m \phi \operatorname{Cos} \alpha-M \frac{\partial \psi}{\partial y} \\
\left(\frac{\partial \psi}{\partial y} \frac{\partial \theta}{\partial x}-\frac{\partial \psi}{\partial x} \frac{\partial \theta}{\partial y}\right)=\frac{1}{\operatorname{Pr}} \frac{\partial^{2} \theta}{\partial y^{2}}+E c\left(\frac{\partial^{2} \psi}{\partial y^{2}}\right)^{2} \\
\left(\frac{\partial \psi}{\partial y} \frac{\partial \phi}{\partial x}-\frac{\partial \psi}{\partial x} \frac{\partial \phi}{\partial y}\right)=\frac{1}{S c} \frac{\partial^{2} \phi}{\partial y^{2}}
\end{gathered}
$$

We now introduce the simplified form of Lie-group transformations namely, the scaling group of transformations (Mukhopadhyay et al. 2005),

$$
\begin{aligned}
\Gamma: x^{*}=x e^{\varepsilon \alpha_{1}}, y^{*}=y e^{\varepsilon \alpha_{2}}, \psi^{*}=\psi e^{\varepsilon \alpha_{3}}, u^{*}=u e^{\varepsilon \alpha_{4}}, \\
v^{*}=v e^{\varepsilon \alpha_{5}}, \theta^{*}=\theta e^{\varepsilon \alpha_{6}}, \phi^{*}=\phi e^{\varepsilon \alpha_{7}}
\end{aligned}
$$

where $\alpha_{1}, \alpha_{2}, \alpha_{3}, \alpha_{4}, \alpha_{5}, \alpha_{6}$ and $\alpha_{7}$ are transformation parameters and $\varepsilon$ is a small parameter.

Equation (15) may be considered as a pointtransformation which transforms co-ordinates $(x, y, \psi, u, v, \theta, \phi)$ to the coordinates $\left(x^{*}, y^{*}, \psi^{*}, u^{*}, v^{*}, \theta^{*}, \phi^{*}\right)$.

Substituting transformations Eq. (15) in Eqs. (12), (13) and (14), we get

$$
\begin{aligned}
& e^{\varepsilon\left(\alpha_{1}+2 \alpha_{2}-2 \alpha_{3}\right)}\left(\frac{\partial \psi^{*}}{\partial y^{*}} \frac{\partial^{2} \psi^{*}}{\partial x^{*} \partial y^{*}}-\frac{\partial \psi^{*}}{\partial x^{*}} \frac{\partial^{2} \psi^{*}}{\partial y^{* 2}}\right) \\
& =e^{\varepsilon\left(3 \alpha_{2}-\alpha_{3}\right)} \frac{\partial^{3} \psi^{*}}{\partial y^{* 3}}+e^{-\varepsilon \alpha_{6}} G r \theta \operatorname{Cos} \alpha \\
& +e^{-\varepsilon \alpha_{7}} G m \phi \operatorname{Cos} \alpha-M e^{\varepsilon\left(\alpha_{2}-\alpha_{3}\right)} \frac{\partial \psi^{*}}{\partial y^{*}} \\
& e^{\varepsilon\left(\alpha_{1}+\alpha_{2}-\alpha_{3}-\alpha_{6}\right)}\left(\frac{\partial \psi^{*}}{\partial y^{*}} \frac{\partial \theta^{*}}{\partial x^{*}}-\frac{\partial \psi^{*}}{\partial x^{*}} \frac{\partial \theta^{*}}{\partial y^{*}}\right) \\
& =\frac{1}{\operatorname{Pr}} e^{\varepsilon\left(2 \alpha_{2}-\alpha_{6}\right)} \frac{\partial^{2} \theta^{*}}{\partial y^{* 2}}+e^{\varepsilon\left(4 \alpha_{2}-2 \alpha_{3}\right)} E c\left(\frac{\partial^{2} \psi^{*}}{\partial y^{* 2}}\right)^{2}
\end{aligned}
$$

$$
\begin{aligned}
& e^{\varepsilon\left(\alpha_{1}+\alpha_{2}-\alpha_{3}-\alpha_{7}\right)}\left(\frac{\partial \psi^{*}}{\partial y^{*}} \frac{\partial \phi^{*}}{\partial x^{*}}-\frac{\partial \psi^{*}}{\partial x^{*}} \frac{\partial \phi^{*}}{\partial y^{*}}\right) \\
& =\frac{1}{S c} e^{\varepsilon\left(2 \alpha_{2}-\alpha_{7}\right)} \frac{\partial^{2} \phi^{*}}{\partial y^{* 2}}
\end{aligned}
$$

The system will remain invariant under the group of transformations $\Gamma$, and we would have the following relations among the parameters, namely

$$
\begin{aligned}
& \alpha_{1}+2 \alpha_{2}-2 \alpha_{3}=3 \alpha_{2}-\alpha_{3}=-\alpha_{6}=-\alpha_{7}=\alpha_{2}-\alpha_{3} \\
& \alpha_{1}+\alpha_{2}-\alpha_{3}-\alpha_{6}=2 \alpha_{2}-\alpha_{6}=4 \alpha_{2}-2 \alpha_{3} \\
& \alpha_{1}+\alpha_{2}-\alpha_{3}-\alpha_{7}=2 \alpha_{2}-\alpha_{7}
\end{aligned}
$$

These

relations

gives $\alpha_{2}=\frac{1}{4} \alpha_{1}=\frac{1}{3} \alpha_{3}, \alpha_{4}=\frac{1}{2} \alpha_{1}, \alpha_{2}=-\frac{1}{4} \alpha_{1}$,

$\alpha_{6}=\alpha_{7}=0$

Thus the set of transformations $\Gamma$ reduce to one parameter group of transformations as

$$
\begin{aligned}
& x^{*}=x e^{\varepsilon \alpha_{1}}, y^{*}=y e^{\varepsilon \frac{\alpha_{1}}{4}}, \psi^{*}=\psi e^{\varepsilon \frac{3 \alpha_{1}}{4}}, \\
& u^{*}=u e^{\varepsilon \frac{\alpha_{1}}{2}}, v^{*}=v e^{-\varepsilon \frac{\alpha_{1}}{4}}, \theta^{*}=\theta, \phi^{*}=\phi
\end{aligned}
$$

Expanding by Tailors method in powers of $\varepsilon$ and keeping terms up to the order $\varepsilon$ we get :

$$
\begin{aligned}
& x^{*}-x=x \varepsilon \alpha_{1}, \quad y^{*}-y=y \varepsilon \frac{\alpha_{1}}{4}, \\
& \psi^{*}-\psi=\psi \varepsilon \frac{3 \alpha_{1}}{4}, u^{*}-u=u \varepsilon \frac{\alpha_{1}}{2}, \\
& v^{*}-v=-v \varepsilon \frac{\alpha_{1}}{4}, \theta^{*}-\theta=0, \phi^{*}-\phi=0
\end{aligned}
$$

The characteristic Equations are :

$$
\begin{aligned}
& \frac{d x}{x \alpha_{1}}=\frac{d y}{y \frac{\alpha_{1}}{4}}=\frac{d \psi}{\psi \frac{3 \alpha_{1}}{4}}=\frac{d u}{u \frac{\alpha_{1}}{2}}=\frac{d v}{-v \frac{\alpha_{1}}{4}} \\
& =\frac{d \theta}{0}=\frac{d \phi}{0}
\end{aligned}
$$

Solving the above Equations, we find the similarity transformations

$$
\eta=x^{-\frac{1}{4}} y, \psi^{*}=x^{\frac{3}{4}} f(\eta), \theta^{*}=\theta(\eta), \phi^{*}=\phi(\eta)
$$

Substituting these values in Eqs. (16) - (18), we finally obtain the system of nonlinear ordinary differential Equations

$$
\begin{aligned}
f^{\prime \prime \prime} & +\frac{3}{4} f f^{\prime \prime}-\frac{1}{2} f^{\prime 2}+G r \theta \operatorname{Cos} \alpha \\
& +G m \phi \operatorname{Cos} \alpha-M f^{\prime}=0 \\
\theta^{\prime \prime} & +\frac{3}{4} \operatorname{Pr} f \theta^{\prime}+\operatorname{Pr} E c f^{\prime \prime 2}=0 \\
\phi^{\prime \prime}+ & \frac{3}{4} S c f \phi^{\prime}=0
\end{aligned}
$$

The corresponding boundary conditions take the form 


$$
\begin{aligned}
& f=0, f^{\prime}=0, \theta=1, \phi=1 \text { at } \eta=0 \\
& f^{\prime} \rightarrow 0, \theta \rightarrow 0, \phi \rightarrow 0 \text { as } \eta \rightarrow \infty
\end{aligned}
$$

\section{NUMERICAL SOLUTION}

The set of nonlinear ordinary differential Eqs. (21) (23) with boundary conditions Eq. (24) have been solved by using the Runge-Kutta fourth order along with Shooting method. First of all, higher order nonlinear differential Eqs. (21) - (23) are converted intosimultaneous linear differential equations of first order and they are further transformed into initial value problem by applying the shooting technique (Jain et al. 1985). The resultant initial value problem is solved by employing Runge-Kutta fourth order technique. The step size $\Delta \eta=0.01$ is used to obtain the numerical solution with five decimal place accuracy as the criterion of convergence. In the next section, the results are discussed in detail.

\section{Result ANd Discussion}

As a result of the numerical calculations, the dimensionless velocity, temperature and concentration distributions for the flow under consideration are obtained and their behavior have been discussed for variations in the governing parameters viz., the thermal Grashof number $G r$, solutal Grashof number $G m$, magnetic field parameter $M$, angle of inclination $\alpha$, Prandtl number $P r$, Eckert number $E c$ and Schmidt number Sc. In the present study following default parameter values are adopted for computations: $G r=G m=2.0, \alpha=30^{\circ}, M=2.0$,

$\operatorname{Pr}=0.71, E c=0.01, S c=0.6$. All graphs therefore correspond to these values unless specifically indicated on the appropriate graph.

In order to assess the accuracy of our computed results, the present result has been compared with Sivasankaran et al. (2006) for different values of $\mathrm{Gr}$ is shown Fig. 2. It is observed that the agreements with the solution of velocity profiles are excellent.

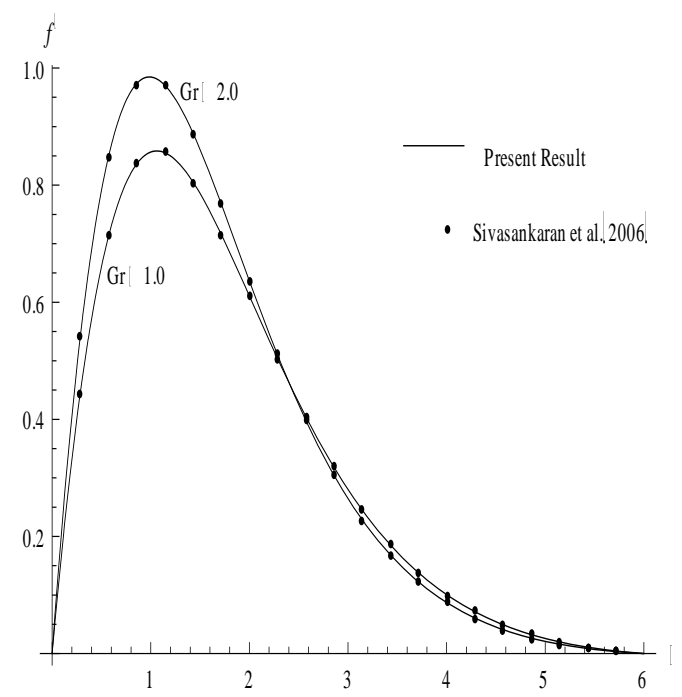

Fig. 2. Comparison of velocity profiles
The influence of the thermal Grashof number $G r$ on the velocity is presented in Fig. 3. The thermal Grashof number $G r$ signifies the relative effect of the thermal buoyancy force to the viscous hydrodynamic force in the boundary layer. As expected, it is observed that there is a rise in the velocity due to the enhancement of thermbuoyancy force. Here, the positive values of $\mathrm{Gr}$ correspond to cooling of the plate. Also, as $G r$ increases, the peak values of the velocity increases rapidly near the porous plate and then decays smoothly to the free stream velocity.

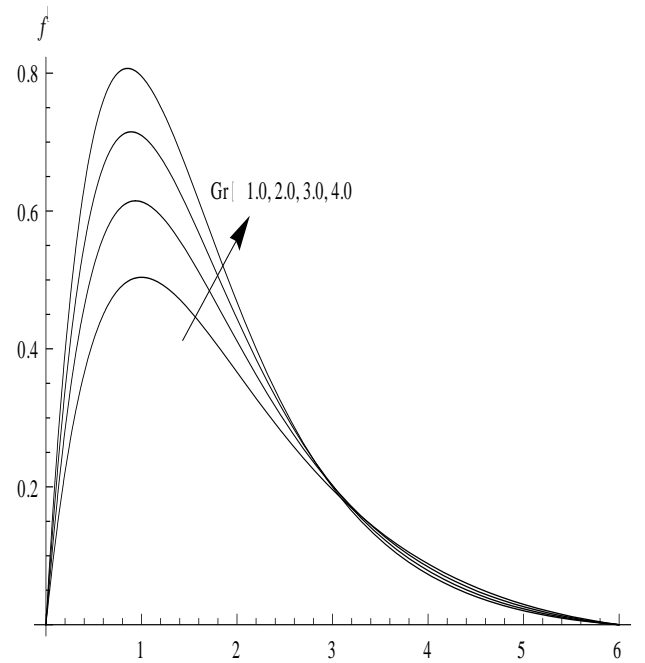

Fig. 3. Velocity profiles for different values of $G r$

Figure 4 presents typical velocity profiles in the boundary layer for various values of the solutal Grashof number $G m$, while all other parameters are kept at some fixed values. The solutal Grashof number $G m$ defines the ratio of the species buoyancy force to the viscous hydrodynamic force. As expected, the fluid velocity increases and the peak value is more distinctive due to increase in the species buoyancy force. The velocity distribution attains a distinctive maximum value in the vicinity of the plate and then decreases properly to approach the free stream value.

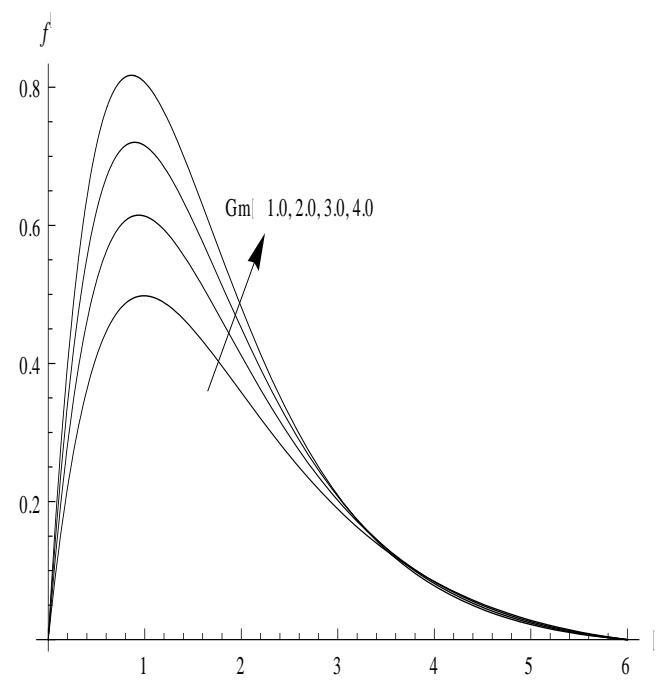

Fig. 4. Velocity profiles for different values of $G m$ 
For various values of the magnetic parameter $M$, the velocity profiles are plotted in Fig. 5. It can be seen that as $M$ increases, the velocity decreases. This result qualitatively agrees with the expectations, since the magnetic field exerts a retarding force on the free convection flow.

Figure 6 shows the effect of angle of inclination to the vertical direction on the velocity profiles. From this figure we observe that the velocity is decreased by increasing the angle of inclination. The fact is that as the angle of inclination increases the effect of the buoyancy force due to thermal diffusion decreases by a factor of $\cos \alpha$. Consequently the driving force to the fluid decreases as a result velocity profiles decrease.

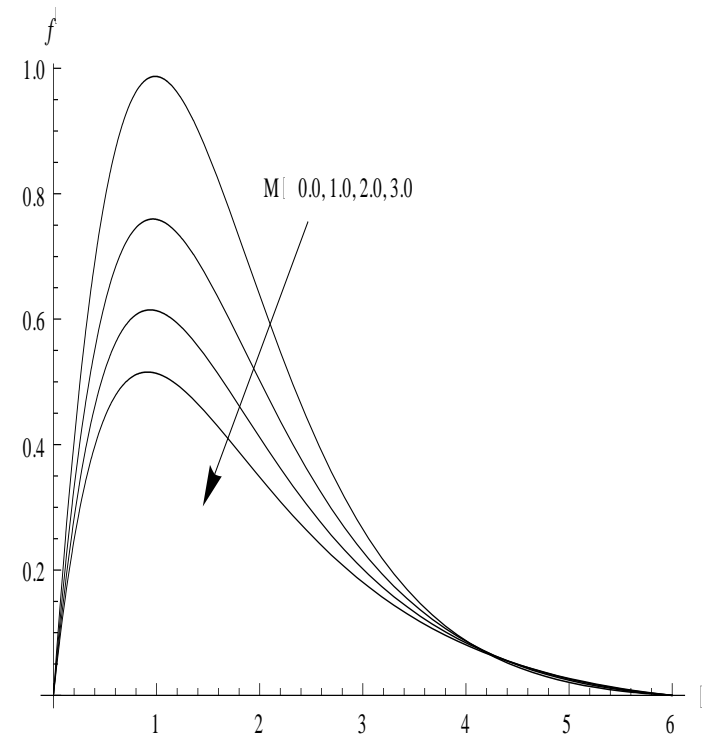

Fig. 5. Velocity profiles for different values of $M$

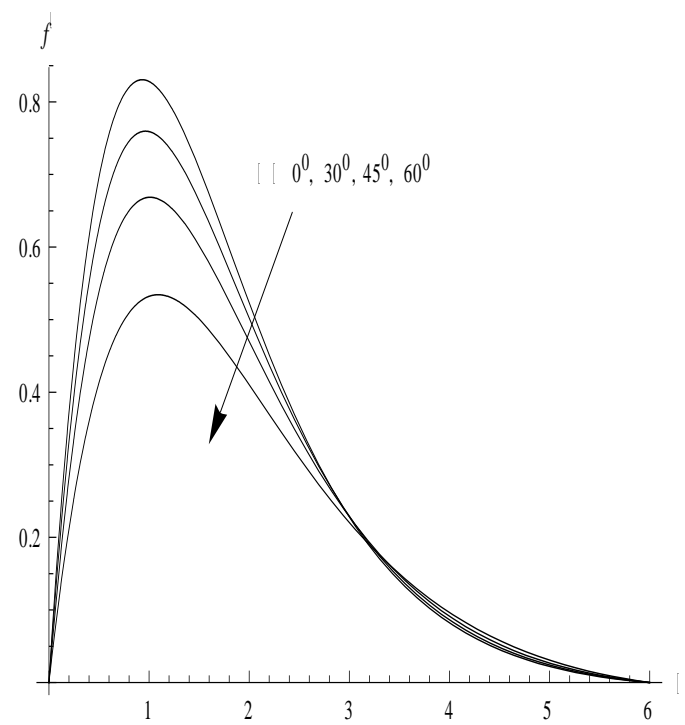

Fig. 6. Velocity profiles for different values of $\alpha$

Figures 7 and 8 illustrate the velocity and temperature profiles for different values of the Prandtl number Pr . The Prandtl number defines the ratio of momentum diffusivity to thermal diffusivity. The numerical results show that the effect of increasing values of Prandtl number results in a decreasing velocity (Fig. 7). From Fig. 8, it is observed that an increase in the Prandtl number results a decrease of the thermal boundary layer thickness and in general lower average temperature within the boundary layer. The reason is that smaller values of $\operatorname{Pr}$ are equivalent to increasing the thermal conductivities, and therefore heat is able to diffuse away from the heated plate more rapidly than for higher values of Pr.Hence in the case of smaller Prandtl numbers as the boundary layer is thicker and the rate of heat transfer is reduced.

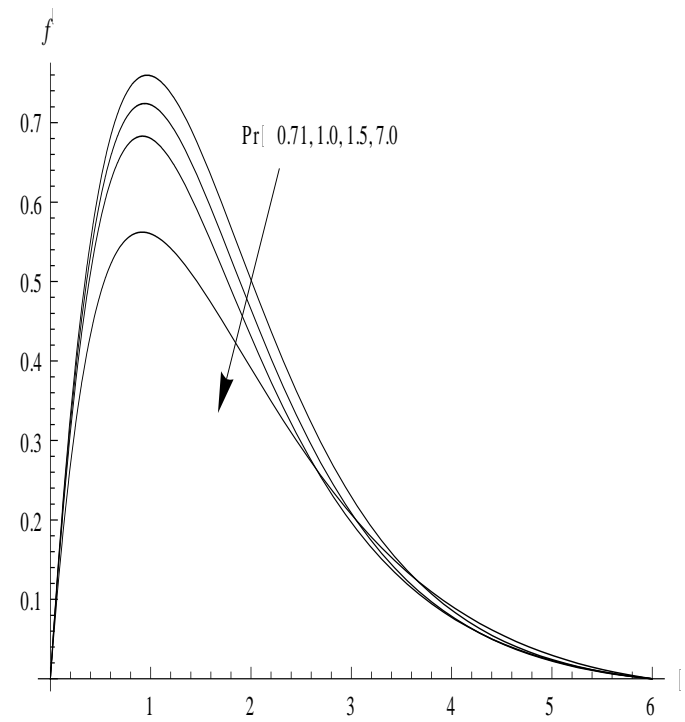

Fig. 7. Velocity profiles for different values of $\operatorname{Pr}$

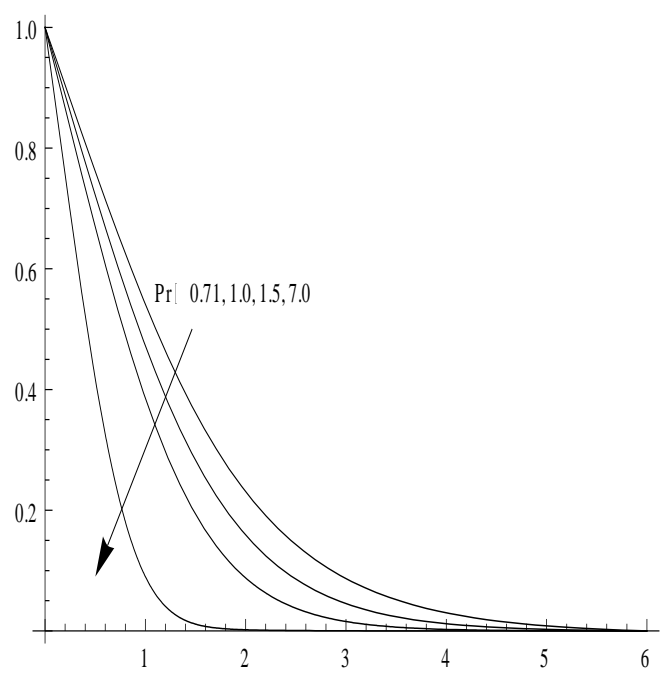

Fig. 8. Temperature profiles for different values of $\mathrm{Pr}$

The effect of the viscous dissipation parameter i.e., the Eckert number $E c$ on the velocity and temperature are shown in Figs 9 and 10 respectively. The Eckert number $E c$ expresses the relationship between the 
kinetic energy in the flow and the enthalpy. It embodies the conversion of kinetic energy into internal energy by work done against the viscous fluid stresses. The positive Eckert number implies cooling of the plate i.e., loss of heat from the plate to the fluid. Hence, greater viscous dissipative heat causes a rise in the temperature as well as the velocity, which is evident from Figs 8 and 9.

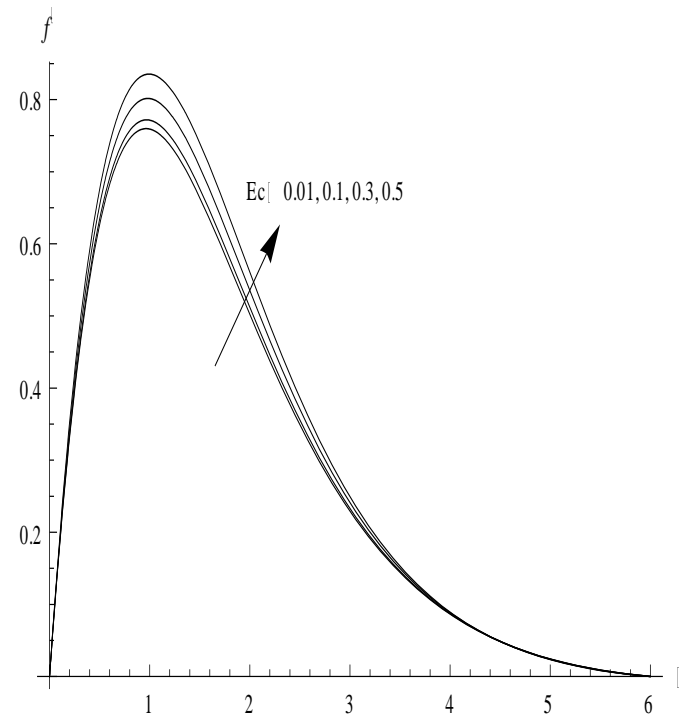

Fig. 9. Velocity profiles for different values of $E_{C}$

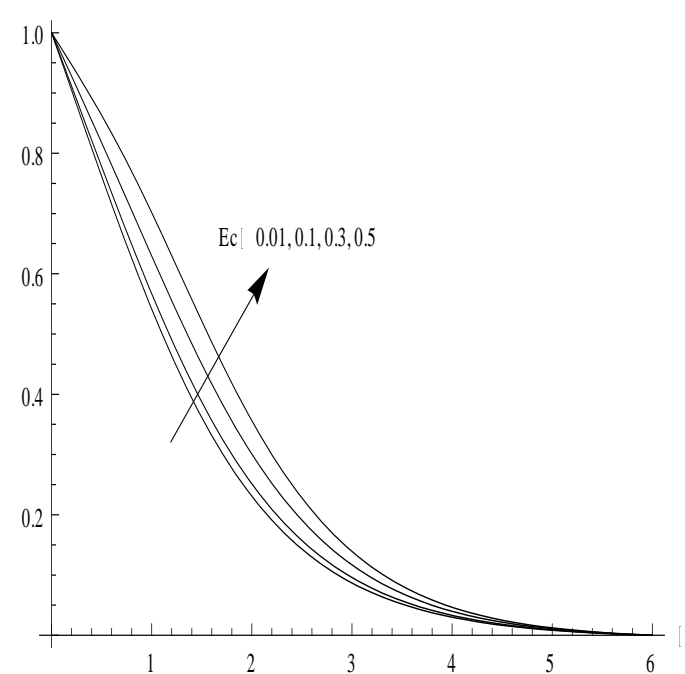

Fig. 10. Temperature profiles for different values of Ec

The influence of the Schmidt number $S c$ on the velocity and concentration profiles are plotted in Figs 11 and 12 respectively. The Schmidt number embodies the ratio of the momentum to the mass diffusivity. The Schmidt number therefore quantifies the relative effectiveness of momentum and mass transport by diffusion in the hydrodynamic (velocity) and concentration (species) boundary layers. As the Schmidt number increases the concentration decreases. This causes the concentration buoyancy effects to decrease yielding a reduction in the fluid velocity. The reductions in the velocity and concentration profiles are accompanied by simultaneous reductions in the velocity and concentration boundary layers. These behaviors are clear from Figs 11 and 12 .

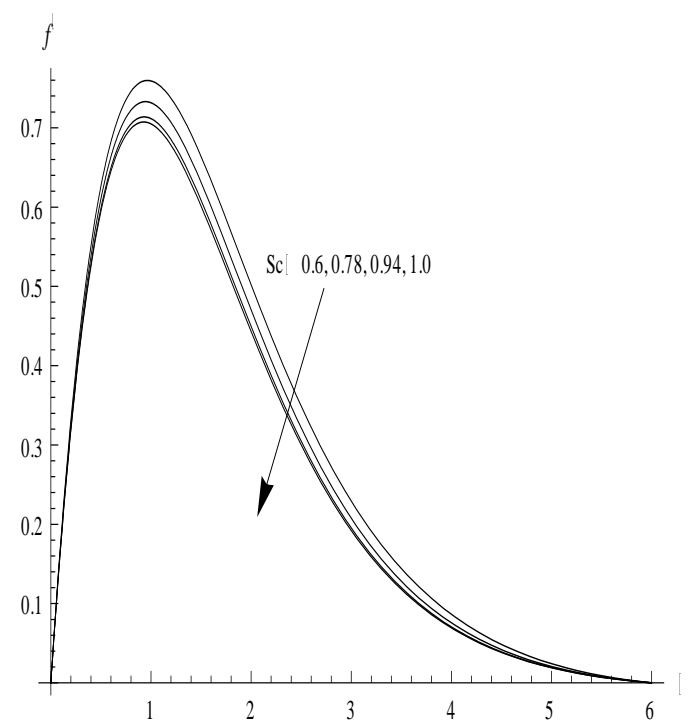

Fig. 11. Velocity profiles for different values of $S c$

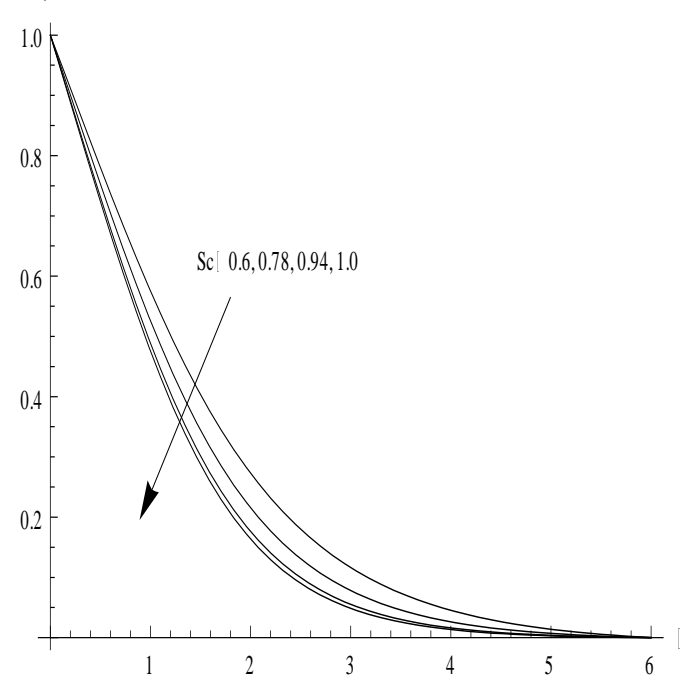

Fig. 12. Concentration profiles for different values of Sc

\section{CONClusion}

With the use of the Lie group analysis, we first find the symmetries of the partial differential equations and then reduce the equations to ordinary differential equations by using scaling and translational symmetries. Exact solutions for translation symmetry and numerical solution for scaling symmetry are obtained. Since the equations are highly nonlinear, a numerical treatment would be more appropriate. From the numerical results, it is predicted that the effect of increasing thermal and solutal Grashof numbers on a viscous incompressible fluid is to increase the flow velocity. It is interesting to note that the temperature of the fluid decreases at a very fast rate in the case of water in comparison with air. 
Therefore, as the Schmidt number increases the concentration decreases. This causes the concentration buoyancy effects to decrease yielding a reduction in the fluid velocity. The analysis has helped engineers understand the mechanisms that are most important in the deposition process. Free convective flow through porous media is an area of research undergoing rapid growth in the fluid mechanics and heat and mass transfer field due to its broad range of scientific and engineering applications. It is associated with petroleum and geothermal processes, fiber and granular insulation materials, high performance insulation buildings, transpiration cooling, packed bed chemical reactors, control of pollutant spread in ground water.

\section{REFERENCES}

Chen, C.H. (2004). Heat and mass transfer in MHD flow by natural convection from a permeable, inclined surface with variable wall temperature and concentration. Acta Mechanica, 172, 219-235.

Elbashbeshy, M.A. (1997). Heat and mass transfer along a vertical plate surface tension and concentration in the presence of magnetic field. Int. J. Eng. Sci., 34(5), 515-522.

Gebhart, B. and J. Mollendorf (1969). Viscous dissipation in external natural convection flows. $J$. Fluid Mech., 38, 97-107.

Gebharat, B. (1962). Effects of viscous dissipation in natural convection. J. Fluid Mech. 14, 225-232.

Gnaneswara Reddy, M, and N. Bhaskar Reddy (2011). Mass transfer and heat generation effects on MHD free convection flow past an inclined vertical surface in a porous medium. Journal of Applied Fluid Mechanics, 4 (3), 7-11.

Gnaneswara Reddy, M, and N. Bhaskar Reddy (2010). Radiation and mass transfer effects on unsteady MHD free convection flow past a vertical porous plate with viscous dissipation. Int. J. Appl. Math and Mech., 6(6), 96-110.

Gnaneswara Reddy, M., and N. Bhaskar Reddy (2010). Soret and dufour effects on steady MHD free convection flow past a semi-infinite moving vertical plate in a porous medium with viscous dissipation, Int. J. Appl. Math and Mech., 6(1), 112.

Helmy, K.A. (1998). MHD unsteady free convection flow past a vertical porous plate. ZAMM, 78, 255270.
Ibrahim, F.S., M.A. Mansour, and M.A.A. Hamad (2005). Lie-group analysis of radiative andmagnetic field effects on free convection and mass transfer flow past a semi-infinite vertical flat plate. Electronic J. Differential Equations, 39, 1-17.

Jain ,M.K., S. R. K. Iyengar, and R.K. Jain (1985). Numerical Methods for Scientific and Engineering Computation. Wiley Eastern Ltd., New Delhi, India.

Kalpakides, V.K. and K.G. Balassas (2004). Symmetry groups and similarity solutions for a free convective boundary-layer problem. Int. J. Non-linear Mech., $39,1659-1670$

Mukhopadhyay, S., G.C. Layek, and S.A. Samad (2005). Study of MHD boundary layer flow over a heated stretching sheet with variable viscosity. Int. J. Heat Mass Transf. 48, 4460-4466.

Pakdemirli, M. (1992). Similarity analysis of compressible boundary layers for arbitrary profiles. Mech. Res. Comm., 19(5), 399-406.

Pakdemirli, M. and M. Yurusoy (1998). Similarity transformations for partial differential Equations. SIAM Rev., 40(1), 96-101.

Sivasankaran, S., M. Bhuvaneswari , P. Kandaswamy, and E.K. Ramasami (2006). Lie group analysis of natural convection heat and mass transfer in an inclined surface. Nonlinear Analysis: Modelling and Control, 11 (1), 201-212.

Soundalgekar, V.M., S.K. Gupta and N.S. Birajdar (1979). Effects of mass transfer and free effects on MHD Stokes problem for a vertical plate. Nucl.Eng.Des., 53, 309-346.

Yurusoy, M. and M. Pakdemirli (1997). Symmetry reductions of unsteady three-dimensional boundary layers of some non-Newtonian Fluids. Int. J. Eng. Sci., 35(8), 731-740.

Yurusoy, M. and M. Pakdemirli (1999). Exact solutions of boundary layer equations of a special non-Newtionian fluid over a stretching sheet. Mech. Res. Comm., 26(2), 171-175.

Yurusoy, M. and M. Pakdemirli (2001). Lie group analysis of creeping flow of a second grade fluid, Int. J. Non-linear Mech., 36, 955-960. 